\title{
Video Article \\ Investigation of Synaptic Tagging/Capture and Cross-capture using Acute Hippocampal Slices from Rodents
}

\author{
Mahesh Shivarama Shetty ${ }^{1,2}$, Mahima Sharma ${ }^{1,2}$, Neo Sin Hui ${ }^{1,2}$, Ananya Dasgupta ${ }^{1,2}$, Suma Gopinadhan ${ }^{1,2}$, Sreedharan Sajikumar ${ }^{1,2}$ \\ ${ }^{1}$ Department of Physiology, Yong Loo Lin School of Medicine, National University of Singapore \\ ${ }^{2}$ Neurobiology/Aging Programme, Life Sciences Institute, National University of Singapore
}

Correspondence to: Sreedharan Sajikumar at phssks@nus.edu.sg

URL: https://www.jove.com/video/53008

DOI: doi: $10.3791 / 53008$

Keywords: Neuroscience, Issue 103, Long-term potentiation, Long-term depression, Synaptic tagging, Synaptic tagging and Capture, Cross-capture Date Published: 9/4/2015

Citation: Shetty, M.S., Sharma, M., Hui, N.S., Dasgupta, A., Gopinadhan, S., Sajikumar, S. Investigation of Synaptic Tagging/Capture and Crosscapture using Acute Hippocampal Slices from Rodents. J. Vis. Exp. (103), e53008, doi:10.3791/53008 (2015).

\section{Abstract}

Synaptic tagging and capture (STC) and cross-tagging are two important mechanisms at cellular level that explain how synapse-specificity and associativity is achieved in neurons within a specific time frame. These long-term plasticity-related processes are the leading candidate models to study the basis of memory formation and persistence at the cellular level. Both STC and cross-tagging involve two serial processes: (1) setting of the synaptic tag as triggered by a specific pattern of stimulation, and (2) synaptic capture, whereby the synaptic tag interacts with newly synthesized plasticity-related proteins (PRPs). Much of the understanding about the concepts of STC and cross-tagging arises from the studies done in CA1 region of the hippocampus and because of the technical complexity many of the laboratories are still unable to study these processes. Experimental conditions for the preparation of hippocampal slices and the recording of stable late-LTP/LTD are extremely important to study synaptic tagging/cross-tagging. This video article describes the experimental procedures to study long-term plasticity processes such as STC and cross-tagging in the CA1 pyramidal neurons using stable, long-term field-potential recordings from acute hippocampal slices of rats.

\section{Video Link}

The video component of this article can be found at https://www.jove.com/video/53008/

\section{Introduction}

The encoding and storage of information in the brain still remains the most significant and keenly pursued challenge in neuroscience. Over the years, long-term potentiation (LTP) and long-term depression (LTD) have emerged as the leading cellular correlates of memory ${ }^{1,2}$. These activity dependent changes, which exhibit input specificity and associativity, result in the stabilization of memory traces in the neuronal networks ${ }_{1,3,4}$. The maintenance of the two forms of synaptic plasticity requires the synthesis of plasticity-related products (PRPs) ${ }^{5-10}$. Synapse specificity that involves the interaction of newly synthesized protein only with specific activated synapses expressing LTP or LTD, is critical to memory. This specificity is explained by the concept of 'Synaptic Tagging and Capture' (STC), where the PRPs interact with recently active, 'tagged' synapses ${ }^{11,12}$. The STC process offers a framework for associative properties of memories at the cellular level. It provides us with a conceptual basis of how short-term forms of plasticity are transformed into long-lasting forms of plasticity in an associative and time-dependent manner ${ }^{13}$.

During the process of STC, a strong tetanization in one input that leads to protein synthesis dependent late-LTP, results in the reinforcement of a protein synthesis independent early-LTP induced in another independent input on to the same population of neurons into a persistent one ${ }^{13}$. The setting of a local synaptic tag by a transient neural activity and the synthesis of the diffusible PRPs by the strong neural activity are the two key events during STC ${ }^{13,14}$. The capture of the PRPs by the recently potentiated 'tagged' synapses is fundamental to the maintenance of long-term potentiation. Many studies have been done to confirm the existence of STC phenomenon ${ }^{15-17}$ and identify the candidate 'tags', ${ }^{\prime 18}$ and 'PRPs', Calcium/calmodulin-dependent protein kinase II (CaMKII) and extracellular signal-regulated kinase1/2 (ERK1/2); CaMKIV, Protein Kinase M (PKM) and brain-derived neurotrophic factor (BDNF) are some of the candidate molecules for 'tag' and 'PRP' respectively ${ }^{19-21}$. The synaptic tagging model has further been expanded to include the positive associative interactions between LTP and LTD - the "synaptic cross-tagging" 22 In synaptic cross-tagging, a late LTP/ LTD in one synaptic input transforms the opposite protein synthesis-independent early-LTD/LTP in an independent input into its long-lasting form or vice versa ${ }^{22}$.

The hippocampal slice preparation is the most widely used model in the studies of long-term synaptic plasticity ${ }^{23,24}$. Much of the understanding about the concepts of synaptic tagging and cross- tagging arises from the studies done in CA1 region of the hippocampus and because of the technical complexity many of the labs are still unable to study these processes. Experimental conditions for the preparation of rat hippocampal slices and the recording of stable late-LTP/LTD for extended hours are extremely important to study synaptic tagging/cross-tagging ${ }^{23,25,26}$. This article describes the detailed experimental procedures for studying long-term plasticity processes such as STC and cross-tagging in the CA1 pyramidal neurons using stable, long-term field-potential recordings from acute hippocampal slices of rats. 
Protocol

All animal procedures were approved by the Institutional Animal Care and Use Committee (IACUC) of National University of Singapore.

\section{Preparation of Artificial Cerebrospinal Fluid (ACSF)}

1. Prepare the ACSF consisting of (in $\mathrm{mM}$ ) $124 \mathrm{NaCl}, 3.7 \mathrm{KCl}, 1.0 \mathrm{MgSO}_{4} .7 \mathrm{H}_{2} \mathrm{O}, 2.5 \mathrm{CaCl}_{2} .2 \mathrm{H}_{2} \mathrm{O}, 1.2 \mathrm{KH}_{2} \mathrm{PO}_{4}, 24.6 \mathrm{NaHCO}_{3}$, and $10 \mathrm{D}$ glucose. Ensure the pH of the ACSF is between 7.2-7.4 when bubbled to saturation with $95 \% \mathrm{O}_{2}$ and $5 \% \mathrm{CO}_{2}$ mixture (carbogen). ${ }^{21}$ Use this ACSF for both the dissection, slice preparation and for perfusion during the electrophysiological recordings.

NOTE: Use clean apparatus for measuring and holding the ACSF. Using unclean apparatus may lead to cloudy solutions or formation of precipitates. Use deionized water for all the preparations.

2. Prepare a $2 \mathrm{~L} 10 x$ ACSF stock excluding $\mathrm{NaHCO}_{3}$ and $\mathrm{D}$-Glucose in a volumetric flask. Add reagents to deionized water in the following order: $\mathrm{NaCl}(144.96 \mathrm{~g}), \mathrm{KCl}(5.52 \mathrm{~g}), \mathrm{MgSO}_{4} .7 \mathrm{H}_{2} \mathrm{O}(4.92 \mathrm{~g}), \mathrm{CaCl}_{2} .2 \mathrm{H}_{2} \mathrm{O}(7.56 \mathrm{~g}), \mathrm{KH}_{2} \mathrm{PO}_{4}(3.28 \mathrm{~g})$ and top up to a volume of $2 \mathrm{~L}$. Stir continuously for at least 30 min using a magnetic stirrer to ensure all reagents are dissolved. Store the stock in $4{ }^{\circ} \mathrm{C}$ and use within 2 weeks.

3. Prior to the dissection and the experiments, dilute the ACSF stock in a volumetric flask along with the addition of required amounts of $\mathrm{NaHCO}_{3}$ and D-glucose. For $1 \mathrm{~L}$ solution, dilute $100 \mathrm{ml}$ of the stock to $1 \mathrm{~L}$ after adding $2.07 \mathrm{~g} \mathrm{NaHCO}$ and $1.802 \mathrm{~g} \mathrm{D}$-glucose. The ACSF should be a clear solution free of any precipitate or undissolved particles.

4. Cool about $200-300 \mathrm{ml}$ of ACSF on ice, to be used during dissection. Ensure that the ACSF used for dissection is between $2-4{ }^{\circ} \mathrm{C}$. Use the remaining ACSF for electrophysiological experiments. Bubble all ACSF solutions to saturation with carbogen $\left(5 \% \mathrm{CO}_{2}, 95 \% \mathrm{O}_{2}\right)$ continuously. While waiting for the ACSF to cool, prepare the dissection area and the slice chamber.

\section{Preparation of Interface Chamber}

NOTE: An interface brain slice chamber, used for incubating the slices and maintaining them during electrophysiological recordings (Figure 2B), consists of two compartments. The lower chamber contains distilled water maintained at $32{ }^{\circ} \mathrm{C}$ by a temperature controller and continuously bubbled with carbogen.

1. Switch on the temperature controller and preset it at $32{ }^{\circ} \mathrm{C}$. Wash the upper chamber for 10 to 15 min by running distilled water through the inflow tubing. Ensure that the upper chamber is clean before placing the net. Check that water level in the bottom chamber is about $70 \%$ filled with distilled water.

2. Place the net in the upper chamber to provide a resting surface for slices (Figure 2C). Adjust the outflow tubing to ensure that the solution level is sufficiently wetting the whole area of the net. Place the lid above the net to maintain a humidified carbogen atmosphere within the upper chamber.

3. Adjust the flow rate to $1 \mathrm{ml} / \mathrm{min}$. Maintain this flow rate throughout the slice incubation period and the experiment. Start carbogenating the freshly prepared 1x ACSF and immerse the inflow tubing into the ACSF. Allow 20 min for the ACSF to be saturated with carbogen and for the upper chamber to be filled with it.

\section{Preparation of Acute Hippocampal Slices}

NOTE: The dissection protocol consists of (1) Removal of brain from the animal into cold ACSF and (2) Isolation and slicing of the hippocampus. In order for neurons to remain viable, isolate and place the brain in cold ACSF quickly and complete the whole process including slicing within 3-5 $\min$.

1. Removal of the brain into cold ACSF

1. Lay out the dissection tools in the manner shown in Figure 1A. Arrange the tools according to the order of use to facilitate the dissection process. Before starting, ensure all the dissection tools are ready.

2. Mount a razor blade, cleaned with ethyl acetate, absolute ethanol and distilled water, onto the manual tissue chopper (Figure 1B), secure it firmly and ensure that the cutting edge is evenly aligned. Test chopping a piece of filter paper to ensure that the blade is firmly secured. Set the sliding Vernier micrometer to its starting position.

3. Euthanize the animal using carbon dioxide $\left(\mathrm{CO}_{2}\right)$ in an induction chamber and decapitate with bandage scissors or Guillotine. Using an Iris scissors, remove the skin and fur above the skull. Make a cut through the posterior to remove the brainstem.Make a small incision along the right side of the skull and a longer incision on the left.

CAUTION! Make only a small incision to the side that is used for the experiments to avoid damaging it. When inserting the scissors, make sure that the force applied is upwards to avoid damage to the brain.

4. Carefully remove the skull with a bone rongeur starting from the left to the right side of skull to reveal the cortex. A thin layer of dura can also be seen. Carefully remove the frontal plates with the rongeur. Remove most of the dura along with the frontal plates. CAUTION! Be careful that the dura does not slice through the brain tissue.

5. Remove the remaining dura, if any, specifically in the junction between cortex and cerebellum with the flat end of a spatula. For steps 3.1.5 and 3.1.6, maintain pressure upwards i.e., away from the brain to avoid damaging it. Using the spatula, gently scoop the brain into a petri dish filled with cold and carbogenated ACSF $\left(2-4^{\circ} \mathrm{C}\right)$, placed on an aluminum cooling block.

2. Isolation of the hippocampus

1. Using a scalpel, make a straight cut to remove the cerebellum and another cut to remove the anterior portion of the brain (approximately one quarter). Make a shallow cut along the midline.

2. Carefully remove the cortex with a sickle scaler, starting from the midline to reveal the dorsal hippocampus. Remove the layer of cortex above the hippocampus. Use fingers or angled forceps to support the brain. Make a small cut to the hippocampal commissure. Gently remove the hippocampus with the sickle scaler starting from the dorsal hippocampus using rolling motions. 
CAUTION! Be gentle to avoid stretching and tearing the hippocampus.

3. Remove any cortex and connective tissues around the isolated hippocampus with the sickle scaler.

3. Slicing the hippocampal tissue and transferring the slices onto the interface chamber

1. Place a piece of ACSF-soaked filter paper (Grade 1, $30 \mathrm{~mm}$ ) on the slicing stage of the manual slicer. Scoop and place the hippocampal tissue onto the filter paper. Move the filter paper to align the hippocampus at a proper orientation in relation to the blade of the slicer so that the hippocampus is sliced at an angle of about $70^{\circ}$ to the fimbria.

2. Blot the excess solution surrounding the hippocampal tissue with a folded filter paper (Grade 1,85 mm) leaving the hippocampus slightly wet. Start slicing the hippocampus transversely. Slice and discard tissue from the extreme end of the hippocampus where the slice morphology is not clear.

3. Slice the remaining tissue into $400 \mu \mathrm{m}$-thick slices. Pick up hippocampal slices gently from the blade with a brush with soft bristles using gentle swiping movements and place the slices into a small beaker filled with cold carbogenated ACSF. Perform the steps 3.3.1-3.3.3 as quickly as possible since the hippocampal tissue is exposed to air. NOTE: Generally two-thirds of the hippocampus is sliced, and 4-6 slices with clear morphology can be prepared.

4. Transfer the slices gently onto the net in the slice chamber using a clean plastic Pasteur pipette with a broad tip (made by cutting away 2-3 cm of the tip). Carefully adjust the position of the slices on the net using a small syringe with a bent tip. Position the slices in a manner that facilitates electrode location and recording. Check to ensure that the slices are sufficiently surrounded by ACSF but are not submerged or floating (Figure 2C-D). Cover the chamber and incubate the slices for 2-3 hr. NOTE: The pyramidal cell layer in the healthy slices should show some transparency.

\section{Recording of CA3-CA1 Synaptic Responses}

NOTE: The electrophysiology set-up used for field potential recording is shown in Figure 2A. A Faraday cage is strongly recommended if the electrical interference is beyond the control after the proper grounding of the electrical settings. Many different types of submerged and interface chambers are commercially available. However, interface chambers are preferred as slices exhibit more robust synaptic responses in them.

1. Positioning of electrodes

1. Turn on the electrical apparatus (stimulators and amplifiers) to be used. Mount and secure the stimulating and recording electrodes in the plexiglass holders of the micromanipulators. NOTE: We use monopolar, lacquer-coated, stainless steel electrodes of $5 \mathrm{M} \Omega$ resistance for both stimulating and recording purposes.

2. Before use, insert these electrodes inside the pulled glass capillaries and secure with epoxy glue exposing only small portion of the electrode tip (Figure 2E). This gives strength to the otherwise slender electrodes and helps to secure them firmly in the electrode holders.

3. Guided under the microscope, position the stimulating electrode(s) in the stratum radiatum of the CA1 region to stimulate the Schaffer collateral fibers and the recording electrode in the apical dendritic region of CA1 to record field-EPSP (fEPSP) responses.

NOTE: Approaching the liquid surface above the slice with the electrodes gives a sound that helps to locate quickly the surface of the slice (provided, the amplifier is connected to a loudspeaker).

4. In synaptic tagging and capture experiments, according to the need of the experiment, position two or three stimulating electrodes (S1, S2 or S3) on either side of the recording electrode to stimulate two or more independent but overlapping inputs. Position the stimulating and recording electrodes about $200 \mu \mathrm{m}$ apart.

5. If necessary, locate another recording electrode in the stratum pyramidale layer for recording population spike (Figure 3A).When both the electrodes have touched the slice, using the acquisition software, give a test stimulation to ensure a proper fEPSP signal. NOTE: We use biphasic, constant-current pulses (impulse duration $0.1 \mathrm{msec} /$ half-wave) for test stimulation.

6. Once a proper fEPSP signal is obtained, carefully lower the electrodes about $200 \mu \mathrm{m}$ deep using fine movement knobs of the manipulators. Allow 20 min for the slices to recover. Test the pathway independence with a paired-pulse facilitation protocol ${ }^{27,28}$.

2. Input-output relation

1. Determine the input-output relation (afferent stimulation vs fEPSP slope) for each input by measuring the slope value at a range of current intensities. Perform this between $20 \mu \mathrm{A}$ to $100 \mu \mathrm{A}$. Then set the stimulation intensity for each input to obtain $40 \%$ of the maximum fEPSP slope. Keep this constant throughout the experiment.

2. After 15-20 min, start recording the baseline. Monitor the fEPSP slope closely during this period and reset the stimulus intensity if the slope fluctuates more than $10 \%$ from the set value and start a new baseline. Record at least $30 \mathrm{~min}$ or $1 \mathrm{hr}$ stable baseline before proceeding.

NOTE: For the test or the baseline stimulation, we use four sweeps of $0.2 \mathrm{~Hz}$ biphasic, constant current pulses ( $0.1 \mathrm{msec}$ per polarity) given every $5 \mathrm{~min}$. An average slope of these four responses is then considered as one repeat. The signals are filtered and amplified by a differential amplifier, digitized using an analog-to-digital converter and monitored online with custom-made software.

3. Induction of LTP/LTD using stimulation protocols

NOTE: Both LTP and LTD have been classified as early and late-LTP/LTD based on the requirements of protein synthesis; the latter requiring translation and/or transcription for its late maintenance [for review see ${ }^{4}$. A variety of electrical stimulation paradigms can specifically induce the different forms of LTP and LTD.

1. WTET: $100 \mathrm{~Hz}, 21$ biphasic constant current pulses $(0.2 \mathrm{msec}$ per phase).

2. STET: Three bursts of 100 pulses for one second $(100 \mathrm{~Hz})$ every $10 \mathrm{~min}$ ( Pulse width $0.2 \mathrm{msec}$ per phase).

3. 900 bursts over a 15 min duration. 1 burst consists of 3 pulses $(0.2 \mathrm{msec}$ width) with an interpulse interval of $50 \mathrm{msec}(20 \mathrm{~Hz})$. The inter burst interval is $1 \mathrm{sec}$ ( total number of pulses 2,700 ). 


\section{Cleaning of Slice Chamber and Perfusion System}

1. After the recording is over, collect the hippocampal slices for further biochemical analysis or else discard appropriately. Turn off the carbogen supply and temperature controller. Wash the carbogen bubbler in distilled water.

2. Clean the net thoroughly with a brush and distilled water. Wash the rig for 15-20 min with distilled water at a higher flow rate. Once in 3-4 days, change the distilled water in the lower compartment of the chamber and also clean the chamber regularly with $3 \%$ hydrogen peroxide solution to avoid fungal growth.

\section{Representative Results}

The described methodology has been used to study long-lasting forms of LTP/LTD and its associative interactions such as synaptic tagging and cross-capture from acute hippocampal slices of adult rats. ${ }^{23}$ This technique has proven effective for experiments with both rats (Wistar) and a variety of mouse strains ${ }^{30,31}$. The methodology has been used successfully for stable LTP recordings of up to 8-12 hr. ${ }^{32}$

The 'tag' set by the weak tetanization of one input (S1) captures the 'PRPs' induced by the strong tetanization of another independent but overlapping input (S2; Figure 3B, filled circles) thereby transforming the otherwise decaying form of LTP (early-LTP) in S1 into a long-lasting one (Figure 3B, open circles) (For comparison of early-LTP induced by WTET see ${ }^{20,33}$ ). The PRPs captured by the weak tetanization set tag need not necessarily come from the STET-induced late-LTP but can also be provided by the SLFS-induced late-LTD. This type of positive associative interaction between LTP and LTD is referred to as 'cross-tagging/capture'. The WTET-induced early-LTP in S1 gets reinforced to late-LTP (Figure 3C, open circles) by capturing the PRPs provided by the SLFS-induced late-LTD in S2 (Figure 3C, filled circles). Statistically significant potentiation or depression was maintained in $\mathrm{S} 1$ and $\mathrm{S} 2$ in both cases when compared to its own baseline (Wilcoxon test; $P<0.05$ ).

For the tag-PRP interaction to occur, the temporal order of the two events (weak-before-strong/strong-before-weak) is not crucial as long as the time window between the two events remains within the range of 30-60 minutes. It would be wise to include a third, independent but overlapping synaptic input and use it as a baseline control to monitor the stability of recordings. The electrical stimulation protocols used to induce early- and late forms of LTP/LTD must be validated in single-input experiments for consistency and reliability before using them in STC experiments. We would also like to emphasize the importance of slice preparation methodology described in the protocol since the success of these experiments relies heavily on the quality of the slices. 


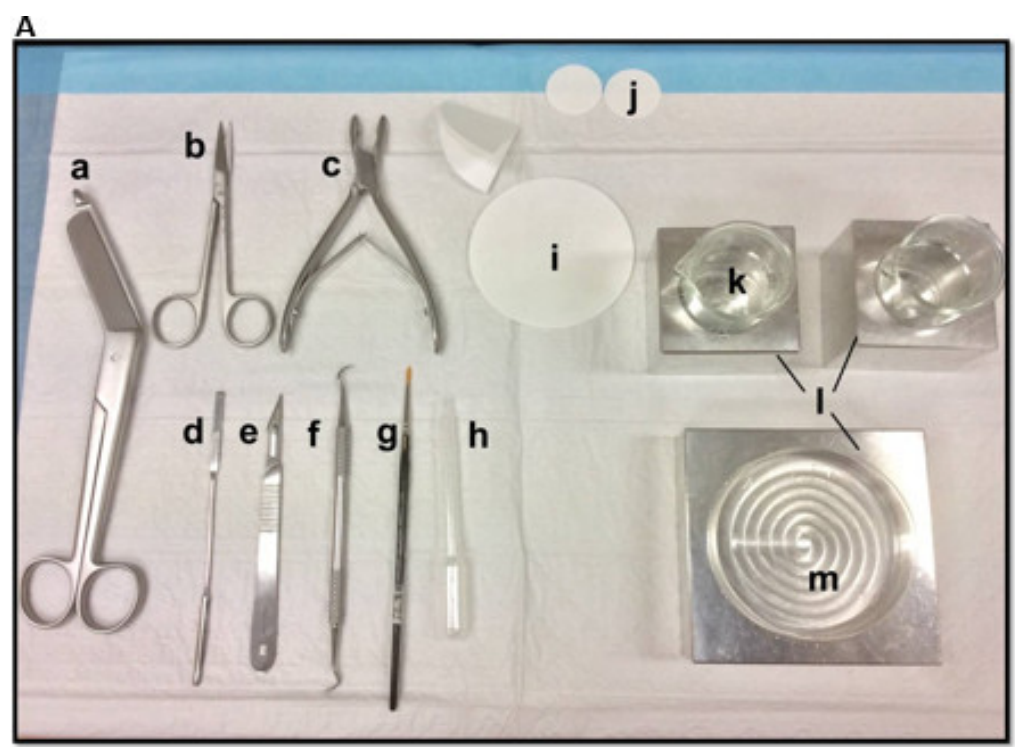

B

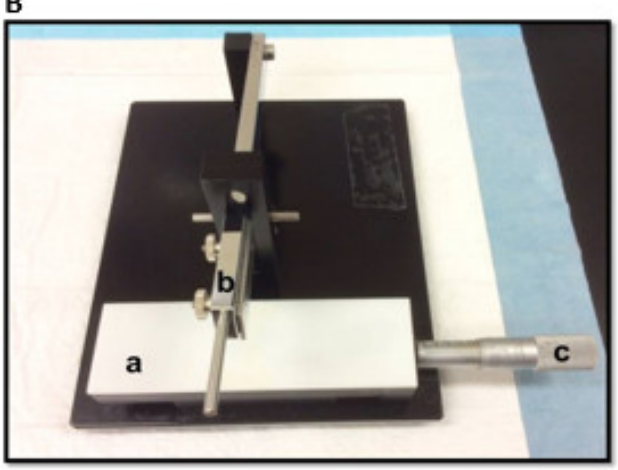

Figure 1. (A) Tools used in the dissection of hippocampus: (a) Bandage Scissors (b) Iris scissors (c) Bone rongeur (d) Thin spatula, (e) Scalpel number 11 (f) Sickle scaler (g) Soft-bristle paint brush (h) Plastic Pasteur pipette (i) filter paper (85mm) (j) filter paper (30mm) (k) Glass beakers (I) Aluminum cooling blocks to fit petri dish and beakers (m) Petri dish. (B) Manual tissue chopper. (a) Platform (b) Cutting arm with blade-holder (c) Vernier micrometer, resolution 10 microns. Please click here to view a larger version of this figure. 
A

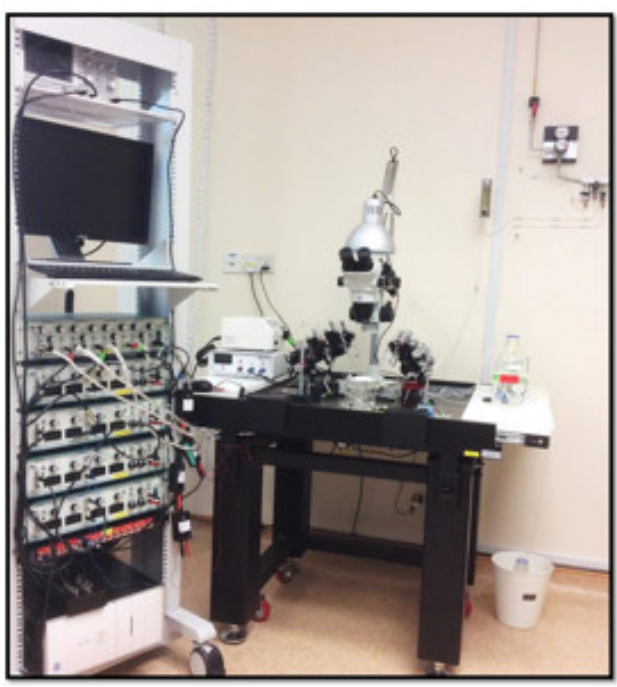

B

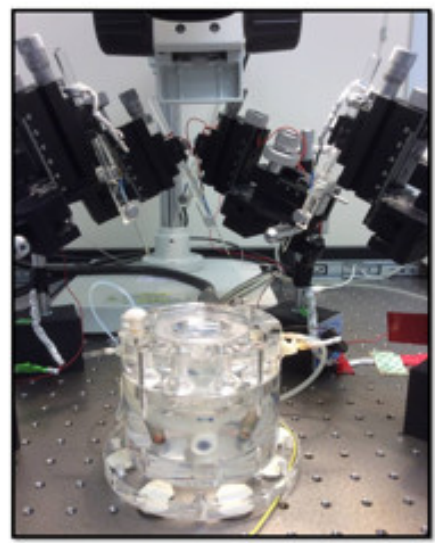

C

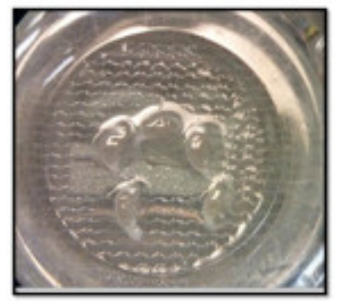

D

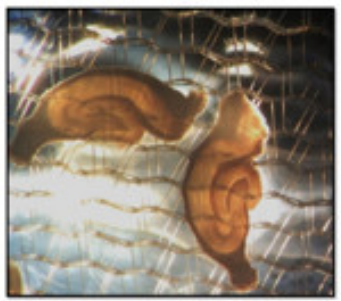

E

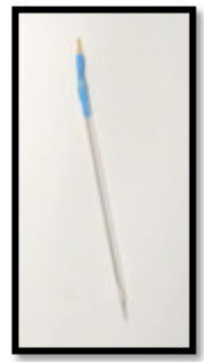

Figure 2. Electrophysiology set-up for field-potential recordings consisting of (A) stimulators (b) a differential amplifier (c) an analog-to-digital converter (d) Oscilloscope (e) computer with acquisition software (f) Vibration-resistant table-top (g) microscope with $>4 x$ magnification (h) interface brain-slice chamber (i) a perfusion system for ACSF and carbogen supply (j) temperature controller (k) an illumination source (I) manipulators with electrode holders. (B) Interface brain-slice chamber. (C) \& (D) Hippocampal slices in the interface chamber. (E) Stainless steel electrode sealed in a glass capillary. Please click here to view a larger version of this figure. 
A

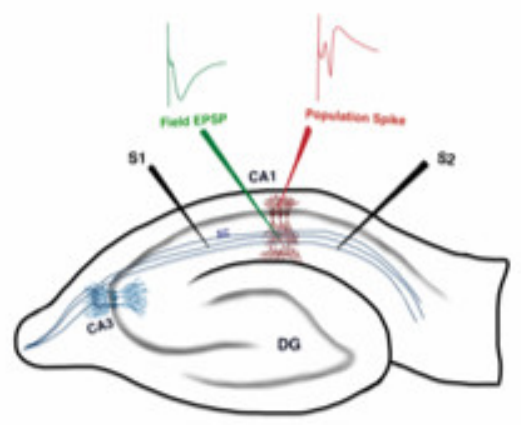

B

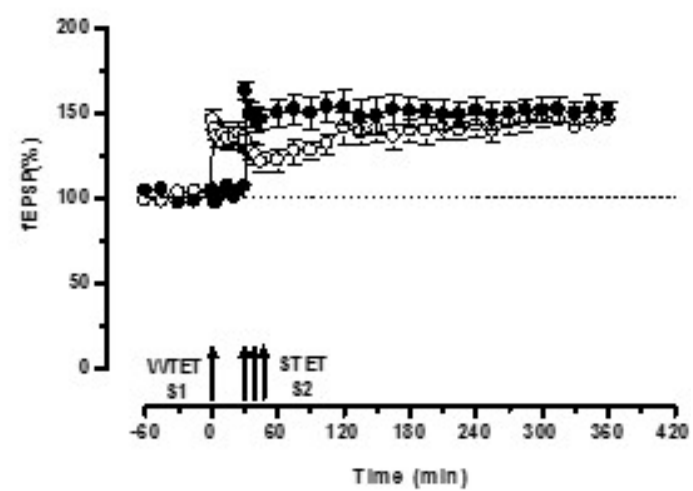

C

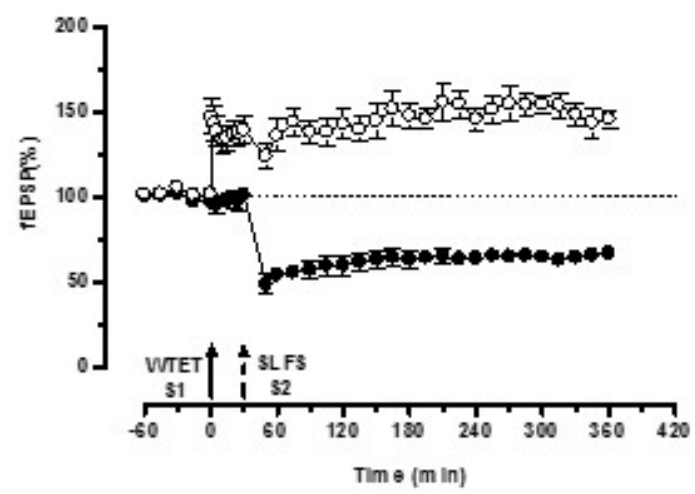

Figure 3. (A) Schematic representation of a transverse hippocampal slice and electrode location for field-potential recording: In this representation, two stimulating electrodes (S1 and S2) are positioned in the stratum radiatum of the CA1 region to stimulate two independent but overlapping synaptic inputs onto CA1 pyramidal neurons. Two extracellular recording electrodes, one to record field-EPSP (excitatory postsynaptic potential) from the apical dendritic compartment and another to record somatic population spike from the pyramidal cell bodies, are located in the stratum radiatum and stratum pyramidale respectively. CA1-cornu ammonis region 1, CA3- cornu ammonis region 3, DG- dentate gyrus, SC- Schaffer collateral fibers, S1- stimulating electrode 1, S2-stimulating electrode 2. (B) Weak before strong paradigm to study STC:

Weak tetanization (WTET) is applied to S1 (open circles) for inducing early-LTP followed by strong tetanization (STET) of S2 (filled circles) at 30 $\mathrm{min}$ to induce late-LTP. The early-LTP in S1 gets reinforced to late-LTP showing tagging and capture interaction $(n=6)$. (C) Weak before strong paradigm to study cross-tagging: Early-LTP is induced by WTET in S1 (open circles) followed by the induction of late-LTD in S2 (filled circles) using SLFS after $30 \mathrm{~min}$. In S1, the early-LTP is transformed to late-LTP lasting $6 \mathrm{hr}$ showing cross-tagging and capture $(\mathrm{n}=6)$. Single arrow represents weak tetanization applied for inducing early-LTP. Triplet of arrows represents strong tetanization for inducing late-LTP. The broken arrow represents the time point at which SLFS was applied to the representative synaptic input to induce late-LTD. Error bars indicate SEM. Please click here to view a larger version of this figure. 


\section{Discussion}

Acute hippocampal slice is an excellent model system for the study of LTP and other functional plasticity processes such as STC and crosscapture. It preserves much of the laminar structural network of the hippocampal circuits, allows precise electrode locations and offers alongside, an open platform for rapid neuropharmacological manipulation without a blood-brain barrier.

This article describes the methodology for the preparation of viable acute hippocampal slices from young adult rats and using them to investigate STC and cross-tagging. Previous research has emphasized that gender and age of the animals are important factors to consider for use in electrophysiology studies. ${ }^{27,28}$ Therefore young adult animals with fully expressed adult receptor functions (male Wistar rats aged 5-7 weeks) are used. ${ }^{23}$ Asymmetries in the connections between the left and right hippocampus have been noted in rodents ${ }^{29}$ and major differences in NMDA receptor expression have been reported as well ${ }^{34}$. We have used the right hippocampus in order to be consistent with our previous LTP studies. ${ }^{23,32}$ However, either of the hippocampi can be used as long as consistency is maintained.

As in any protocol, it is very crucial to perform the isolation and slicing procedures quickly but taking care that the tissue is not stretched, damaged, rendered dry or hypoxic. The variations in $\mathrm{pH}$, temperature and ionic composition of the solutions can have profound effect on the viability of the slices and the results. Hence such variations should be avoided. It has been observed that glutamate receptor-dependent calcium release occurring during the preparation steps can irreversibly affect protein synthesis in nervous tissue ${ }^{35,36,37}$. Using manual tissue slicers can help to minimize this by allowing the process to be completed very quickly as compared to vibraslicers. However, many laboratories also effectively use vibraslicers with necessary precautions to preserve slice viability. Another important factor to consider is the long incubation period before starting the experiments. This has been noted to be really crucial to achieve stability in metabolic state and kinase activation levels in the slices after the disturbance caused during preparation ${ }^{23}$. Such stability is necessary for consistency in long-term recordings. We reemphasize on this observation and suggest the long incubation hours of about $3 \mathrm{hr}$.

A variety of stimulation parameters are known to induce LTP, but the molecular mechanisms elicited in each case may not be the same (for review see ${ }^{38}$ ). This can influence the durability and other characteristics of the LTP which, in turn, can affect the results of synaptic tagging and capture experiments. Hence it is important to validate the stimulation paradigms and characteristics of the elicited LTP under the conditions of the performing laboratory and maintain consistency.

We generally do not consider experiments with very large presynaptic fiber volleys and with maximal fEPSPs less than $0.5 \mathrm{mV}$ and the experiments involving substantial changes in the fiber volley during the recordings are also rejected. Further, while performing two-pathway or three-pathway experiments, it is important to ensure the pathway independence. This can be carried out with a paired-pulse facilitation protocol $^{28}$.

One downside of the interface recording systems is the formation of condensation droplets on the electrodes during the long recording hours due to the temperature and humidity differences between the chamber and the surroundings. These droplets need to be carefully blotted from time to time. Otherwise the droplets can drip onto the slices and cause disturbance or even loss of signals. We usually tackle this by skilfully blotting the droplets guided under the microscope using a slender filter paper wick, without touching the electrodes. However, the best solution would be to use a centralized heating system, such as the ETC system developed by University of Edinburgh researchers.

On a concluding note, a variety of methodologies exist in the laboratories worldwide that are used for the preparation of hippocampal slices for different experimental purposes. Each of the procedure offers some advantages over the other. One needs to carefully optimize the minute details of the protocol to suit the purpose of the experiment. We hope that this article helps in improving some aspects of the methodology for studying late-associative processes such as STC and cross-capture.

\section{Disclosures}

Open access for this video article is sponsored by Cerebos Pacific Limited.

\section{Acknowledgements}

This video article is sponsored by Cerebos Pacific Limited. This work is supported by National Medical Research Council Collaborative Research Grant (NMRC-CBRG-0041/2013) and Ministry of Education Academic Research Funding (MOE AcRF- Tier 1 - T1-2012 Oct -02).

\section{References}

1. Bliss, T. V. P., Collingridge, G. L. A synaptic model of memory: long-term potentiation in the hippocampus. Nature. 361, 31-39 (1993).

2. Siegelbaum, S. A., Kandel, E. R. Learning-related synaptic plasticity: LTP and LTD.. Current Opinion in Neurobiology. 1, 113-120 (1991).

3. Martin, S. J., Grimwood, P. D., Morris, R. G. Synaptic plasticity and memory: an evaluation of the hypothesis. Annual review of neuroscience. 23, 649-711 (2000).

4. Malenka, R. C., Bear, M. F. LTP and LTD: An Embarrassment of Riches. Neuron. 44, 5-21 (2004).

5. Krug, M., Lossner, B., Ott, T. Anisomycin blocks the late phase of long-term potentiation in the dentate gyrus of freely moving rats. Brain research bulletin. 13, 39-42 (1984).

6. Frey, U., Krug, M., Reymann, K. G., Matthies, H. Anisomycin blocks an inhibitor of protein synthesis, blocks late phases of LTP phenomena in the hippocampal CA1 region in vitro. Brain research. 452, 57-65 (1988).

7. Otani, S., Marshall, C. J., Tate, W. P., Goddard, G. V., Abraham, W. C. Maintenance of long-term potentiation in rat dentate gyrus requires protein synthesis but not messenger RNA synthesis immediately post-tetanization. Neuroscience. 28, 519-526 (1989). 
8. Frey, U., Frey, S., Schollmeier, F., Krug, M. Influence of actinomycin D, a RNA synthesis inhibitor, on long-term potentiation in rat hippocampal neurons in vivo and in vitro. The Journal of physiology. 490, (3), 703-711 (1996).

9. Nguyen, P. V., Kandel, E. R. A macromolecular synthesis-dependent late phase of long-term potentiation requiring cAMP in the medial perforant pathway of rat hippocampal slices). The Journal of neuroscience : the official journal of the Society for Neuroscience. 16, 3189-3198 (1996).

10. Manahan-Vaughan, D., Kulla, A., Frey, J. U. Requirement of translation but not transcription for the maintenance of long-term depression in the CA1 region of freely moving rats. The Journal of neuroscience : the official journal of the Society for Neuroscience. 20, 8572-8576 (2000).

11. Frey, U., Morris, R. G. M. Synaptic tagging and long-term potentiation. Nature. 385, 533-536 (1997).

12. Frey, U., Morris, R. G. Weak before strong: dissociating synaptic tagging and plasticity-factor accounts of late-LTP. Neuropharmacology. $\mathbf{3 7}$, 545-552 (1998).

13. Redondo, R. L., Morris, R. G. Making memories last: the synaptic tagging and capture hypothesis. Nature reviews. Neuroscience. 12, 17-30 (2011).

14. Martin, K. C., Kosik, K. S. Synaptic tagging -- who's it?. Nature reviews. Neuroscience. 3, 813-820 (2002).

15. Frey, U., Morris, R. G. Synaptic tagging: implications for late maintenance of hippocampal long-term potentiation. Trends in neurosciences. 21, 181-188 (1998).

16. Martin, K. C. Synaptic tagging during synapse-specific long-term facilitation of Aplysia sensory-motor neurons. Neurobiology of learning and memory. 78, 489-497 (2002).

17. Shires, K. L., Da Silva, B. M., Hawthorne, J. P., Morris, R. G., Martin, S. J. Synaptic tagging and capture in the living rat. Nature communications. 3, (2012).

18. Ramachandran, B., Frey, J. U. Interfering with the actin network and its effect on long-term potentiation and synaptic tagging in hippocampal CA1 neurons in slices in vitro. The Journal of neuroscience : the official journal of the Society for Neuroscience. 29, 12167-12173 (2009).

19. Sajikumar, S., Navakkode, S., Frey, J. U. Identification of compartment- and process-specific molecules required for 'synaptic tagging' during long-term potentiation and long-term depression in hippocampal CA1. The Journal of neuroscience : the official journal of the Society for Neuroscience. 27, 5068-5080 (2007).

20. Sajikumar, S., Navakkode, S., Sacktor, T. C., Frey, J. U. Synaptic tagging and cross-tagging: the role of protein kinase Mzeta in maintaining long-term potentiation but not long-term depression. The Journal of neuroscience : the official journal of the Society for Neuroscience. 25, 5750-5756 (2005).

21. Redondo, R. L., et al. Synaptic Tagging and Capture: Differential Role of Distinct Calcium/Calmodulin Kinases in Protein SynthesisDependent Long-Term Potentiation. The Journal of Neuroscience. 30, 4981-4989 (2010).

22. Sajikumar, S., Frey, J. U. Late-associativity, synaptic tagging, and the role of dopamine during LTP and LTD. Neurobiology of learning and memory. 82, 12-25 (2004).

23. Sajikumar, S., Navakkode, S., Frey, J. U. Protein synthesis-dependent long-term functional plasticity: methods and techniques. Current Opinion in Neurobiology. 15, 607-613 (2005).

24. Villers, A., Ris, L. Improved preparation and preservation of hippocampal mouse slices for a very stable and reproducible recording of longterm potentiation. Journal of visualized experiments : JoVE. (2013).

25. Pohle, W., Reymann, K., Jork, R., Malisch, R. The influence of experimental conditions on the morphological preservation of hippocampal slices in vitro. Biomedica biochimica acta. 45, 1145-1152 (1985).

26. Reymann, K. G., et al. The duration of long-term potentiation in the CA1 region of the hippocampal slice preparation. Brain research bulletin. 15, 249-255 (1985).

27. Sajikumar, S., Korte, M. Different compartments of apical CA1 dendrites have different plasticity thresholds for expressing synaptic tagging and capture. Learning \& Memory. 18, 327-331 (2011).

28. Li, Q., et al. Making Synapses Strong: Metaplasticity Prolongs Associativity of Long-Term Memory by Switching Synaptic Tag Mechanisms. Cerebral Cortex. 24, 353-363 (2014).

29. Sajikumar, S., Frey, J. U. Anisomycin inhibits the late maintenance of long-term depression in rat hippocampal slices in vitro. Neuroscience Letters. 338, 147-150 (2003).

30. Ishikawa, Y., Tamura, H., Shiosaka, S. Diversity of neuropsin (KLK8)-dependent synaptic associativity in the hippocampal pyramidal neuron. The Journal of physiology. 589, 3559-3573 (2011).

31. Ishikawa, Y., Horii, Y., Tamura, H., Shiosaka, S. Neuropsin (KLK8)-dependent and -independent synaptic tagging in the Schaffer-collateral pathway of mouse hippocampus. The Journal of neuroscience : the official journal of the Society for Neuroscience. 28, 843-849 (2008).

32. Sajikumar, S., Morris, R. G. M., Korte, M. Competition between recently potentiated synaptic inputs reveals a winner-take-all phase of synaptic tagging and capture. Proceedings of the National Academy of Sciences. 111, 12217-12221 (2014).

33. Navakkode, S., Sajikumar, S., Frey, J. U. The type IV-specific phosphodiesterase inhibitor rolipram and its effect on hippocampal long-term potentiation and synaptic tagging. The Journal of neuroscience : the official journal of the Society for Neuroscience. 24, 7740-7744 (2004).

34. Kohl, M. M., et al. Hemisphere-specific optogenetic stimulation reveals left-right asymmetry of hippocampal plasticity. Nature. 14, 1413-1415 (2011).

35. Frey, U., Huang, Y. Y., Kandel, E. R. Effects of cAMP Simulate a Late Stage of LTP in Hippocampal CA1 Neurons. Science. 260, 1661-1664 (1993).

36. Erdogdu, G., Uto, A., Hossmann, K. -A. The effect of global ischemia and recirculation of rat brain on protein synthesis in vitro. Metab Brain Dis. 8, 199-206 (1993).

37. Djuricic, B., Berger, R., Paschen, W. Protein synthesis and energy metabolism in hippocampal slices during extended ( 24 hours) recovery following different periods of ischemia. Metab Brain Dis. 9, 377-389 (1994).

38. Park, P., et al. NMDA receptor-dependent long-term potentiation comprises a family of temporally overlapping forms of synaptic plasticity that are induced by different patterns of stimulation. Philosophical Transactions of the Royal Society B: Biological Sciences. 369, 20130131 (2014). 